\title{
Aplicação do algoritmo de enxame de partículas na otimização do custo energético de bombas de velocidade variável
}

\section{Application of the particle swarm algorithm in the optimization of the energy cost of variable speed pumps}

Data de entrada: 29/04/2020

Data de aprovação: 24/09/2020
José Nilton de Abreu Costa ${ }^{1 *} \mid$ Marco Aurélio Holanda de Castro ${ }^{2} \mid$ Luís Henrique Magalhães Costa ${ }^{1}$ J João Marcelo Costa Barbosa ${ }^{2}$

ORCID ID

Costa JNA (iD https://orcid.org/0000-0001-9691-8067

Castro MAH (D) https://orcid.org/0000-0001-5134-7213
DOI: https://doi.org/10.36659/dae.2022.018
Costa LHM (D) https://orcid.org/0000-0002-1781-4188

Barbosa JMC (D) https://orcid.org/0000-0003-0559-0516

\section{Resumo}

As estratégias de operação dos sistemas de abastecimento de água (SAA) objetivam o abastecimento público ininterrupto, muito embora comumente negligenciem a oscilação da tarifa energética diária. Isso proporciona um acréscimo significativo no custo energético das bombas. Entretanto, além de utilizar eficientemente a tarifa, é necessário considerar também o vasto horizonte de combinações exequíveis de estratégias operacionais, a demanda hídrica variante e a manutenção dos níveis de água nos reservatórios e das pressões nas extremidades dentro de intervalos preestabelecidos. Neste cenário, realizou-se este trabalho, cujo objetivo é determinar perfis diários de velocidade das bombas de um SAA, objetivando a minimização do custo energético diário. Para isso, desenvolveu-se um modelo computacional que opera acoplado ao simulador hidráulico EPANET. O modelo fundamenta-se na técnica meta-heurística PSO (Otimização por Enxame de Partículas), sendo a variável de decisão a velocidade das bombas. Essa técnica procura por estratégias com custo energético reduzido, enquanto o EPANET analisa o desempenho hidráulico. Os resultados evidenciaram a eficácia do PSO na determinação de uma quantidade satisfatória de estratégias de operação e na redução do tempo demandado nessa tarefa. Assim, considera-se que a metodologia desenvolvida pode auxiliar a operação de SAAs, visando à otimização energética.

Palavras-chave: Bombas de rotação variável. Eficiência energética. Otimização.

\section{Abstract}

The strategies of operation of the water supply systems (WSS) aim at the uninterrupted public supply, although they commonly neglect the oscillation of the daily energy tariff. This provides a significant increase in the energy cost of the pumps. However, in addition to efficiently using the tariff, it is also necessary to consider the broad horizon of feasible combinations of operational strategies, water varying demand and the maintenance of water levels in the reservoirs and the pressure in the extremities within pre-established intervals. In this scenario, this work was carried out, whose objective is to determine daily speed profiles of the pumps of an SAA, aiming at minimizing the

\footnotetext{
${ }^{1}$ Universidade Estadual Vale do Acaraú - Sobral - Ceará - Brasil.

2 Universidade Federal do Ceará - Fortaleza - Ceará - Brasil.

* Autor correspondente: nilton_dehaayahoo.com.br.
} 
daily energy cost. For this, a computational model was developed that operates in conjunction with the EPANET hydraulic simulator. The model is based on the meta-heuristic technique PSO (Particle Swarm Optimization), being the decision variable the speed of the pumps. This technique looks for strategies with reduced energy costs while EPANET analyzes hydraulic performance. The results evidenced the effectiveness of the PSO in determining a satisfactory amount of operating strategies and reducing the time required in this task. Thus, it is considered that the methodology developed can help the operation of WSSs, aiming at energy optimization.

Keywords: Variable rotation pumps. Energy efficiency. Optimization.

\section{INTRODUÇÃO}

A conservação de energia, especialmente a de natureza elétrica, é algo a que se dedica a atenção do mundo moderno, em virtude da sua ligação peculiar com questões econômicas e ambientais. Diante do fato de que o setor que mais consome energia elétrica é o industrial e que uma parte significante dessa energia é designada para a alimentação dos seus sistemas motrizes, esses sistemas têm sido considerados alvos principais das medidas que visam a garantir a economia de energia elétrica (SCHMIDLIN JR. et al., 2006).

Em sistemas de abastecimento de água, os gastos relacionados com a operação das bombas hidráulicas podem ser mitigados com a diminuição do consumo de energia. Uma forma conveniente que pode ser adotada para o alcance desse intento é a utilização de bombas de rotação variável (BRV) no lugar daquelas que trabalham com velocidade constante (MARCHI; SIMPSON; ERTRUGUL, 2012). As bombas de rotação variável são instrumentos acoplados a um motor que é regulado por um inversor de frequência, cujo propósito é oscilar a fonte de alimentação, provocando a variação da velocidade rotativa da bomba e, assim, induzi-la a trabalhar com a maior eficiência possível.

O inversor de frequência, por meio do controle da velocidade de rotação do motor, promove a alteração da curva da bomba e mantém constante a curva do sistema (curva da instalação). Isso faz com que o consumo de energia seja proporcional à velocidade rotativa da bomba, ou seja, nem mais nem menos, apenas a quantidade necessária (RODRIGUES, 2007).

No presente contexto, as estratégias de otimização alcançam um significativo papel, pois são capazes de atuar melhorando o desempenho de vários processos, minimizando os custos e maximizando a produção, entre outras vantagens. 0 processo de otimização consiste na busca pela melhor solução para um determinado problema e é composto por uma ou mais funções-objetivo e uma sequência de variáveis de decisão, cujos valores otimizados são o que se pretende determinar, de tal modo que possam ser compatíveis com possíveis restrições pré-estabelecidas (KURITZA et al., 2017).

O problema de otimizar o funcionamento das bombas de uma rede de distribuição de água implica em algumas questões relacionadas ao dimensionamento ótimo da rede e à escolha da configuração ideal do funcionamento das bombas. Nesse sentido, a utilização de bombas de rotação variável torna-se uma técnica oportuna, pois proporciona a adequação entre a quantidade de água bombeada e a demandada pelos consumidores finais.

Nesse cenário, realizou-se este trabalho, cujo objetivo é determinar perfis diários de velocidade das bombas de um SAA, objetivando a minimização do custo energético diário. 
Diante do exposto, o objetivo geral do presente trabalho é determinar configurações diárias de velocidade das bombas de um SAA, objetivando a minimização do custo energético diário. Para a realização deste trabalho, desenvolveu-se uma ferramenta computacional que atua de maneira acoplada ao simulador hidráulico EPANET, simulando o comportamento de bombas de rotação variável. 0 modelo executa a rotina do algoritmo de otimização por enxame de partículas (PSO) para promover a alteração dos valores de velocidade das bombas ao longo do dia, de modo a encontrar uma solução de custo energético ótimo.

\section{SISTEMAS DE BOMBEAMENTO COM INVERSOR DE FREQUÊNCIA}

Num sistema hidráulico, a transformação de energia é feita por meio de bombas e motores hidráulicos. A característica geral de operação é, na unidade de conversão primária, a transformação de energia mecânica em energia hidráulica, que é transmitida pelo fluido até a unidade de conversão secundária, onde é novamente transformada em energia mecânica para a produção de trabalho útil (LINSINGEN, 2001).

Baptista e Coelho (2010) caracterizam as bombas hidráulicas como máquinas que promovem a transformação da energia mecânica que recebem dos motores em energia hidráulica, viabilizando o transporte de fluidos a grandes distâncias e elevadas alturas, que atendem desde as pequenas instalações hidráulicas prediais até os grandes sistemas de irrigação, abastecimento de água, entre outros complexos de grande porte.
De acordo com Kuritza (2017), para que as medidas de planejamento e gestão dos recursos hídricos e energéticos nos sistemas de bombeamento de água possam ser mais bem aplicadas, é importante que se tenha o prévio conhecimento de alguns elementos, tais como a infraestrutura existente, os procedimentos operacionais, os cadastros comerciais e, ainda, que haja monitoramento dos parâmetros elétricos, hidráulicos e mecânicos envolvidos. Avaliar a eficiência energética é fundamental para que sejam estabelecidas prioridades de intervenção e para que seja controlada a eficácia das medidas implementadas.

Entretanto, a aplicação das medidas cabíveis à gestão dos SAAs não configura uma atividade trivial. A elevada complexidade da gestão de sistemas de abastecimento torna necessária a introdução de equipamentos que possam implicar em flexibilidade de operação, permitindo adequar o modo de funcionamento à disponibilidade de recursos e às necessidades dos consumidores, com grande índice de eficiência (ALEGRE et al., 2005).

De acordo com Reis (2010), as bombas são equipamentos aos quais está associada a mais significativa parcela do consumo de energia elétrica na grande maioria das instalações industriais. Em muitas situações, tais instrumentos são comumente ligados por meio de motores de indução trifásicos, que são máquinas potentes e de alta eficiência. A Fig. 1 apresenta o esquema de uma estação de elevatória composta por uma bomba que, funcionando acoplada a um inversor de freqüência, pode operar com velocidade de rotação variável. 


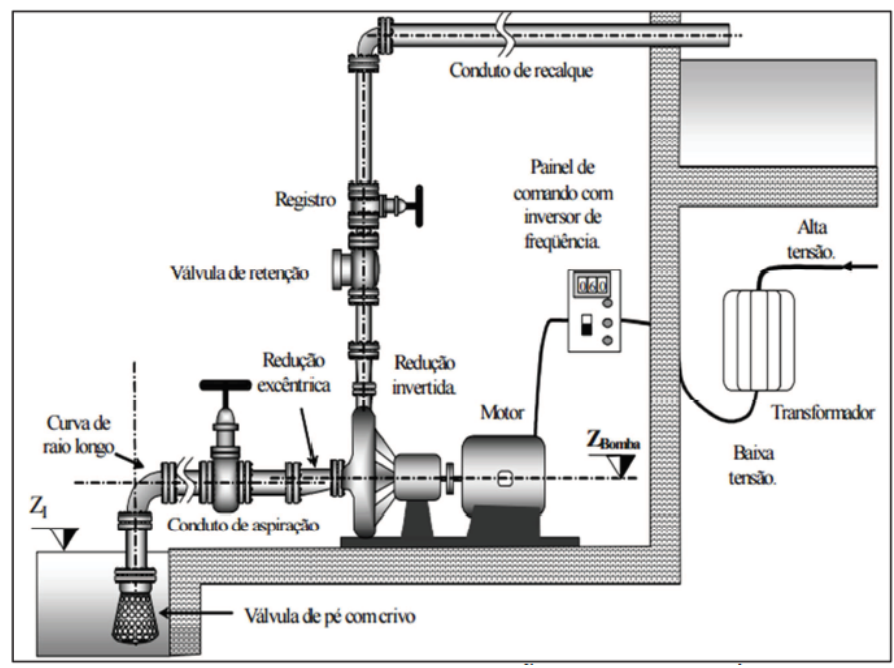

Figura 1 - Sistema de bombeamento com inversor de frequência. Fonte: Borges (2011).

Conforme o exposto, a alteração na velocidade rotativa implica no deslocamento do ponto de operação da bomba, acarretando mudanças nos valores de vazão, altura manométrica, potência e rendimento. Assim, há uma necessidade de estimar, com a máxima acurácia possível, o rendimento da bomba nas suas novas condições de funcionamento, uma vez que este está diretamente relacionado com o consumo de energia do sistema.

Para realizar o cálculo do rendimento final $\eta_{2}$ da bomba, comumente é necessário que se conheçam as coordenadas do ponto de operação final e a equação da curva de rendimento para a rotação nominal. No entanto, como muitas vezes não se dispõe de tais informações, pode-se fazer uma estimativa do rendimento final a partir da Eq. 1, proposta por Costa et al. (2018), em que $\eta$ é o rendimento, $R$ é a razão entre as velocidades de rotação $\left(\mathrm{N}_{2} / \mathrm{N}_{1}\right)$ e os subscritos 1 e 2 se referem às rotações inicial e final, respectivamente.

$\eta_{2}=\eta_{1} \cdot(2-R)^{[0,4 \ln R]}$

Ressalta-se que a rotação inicial $N_{1}$ é um valor de velocidade para o qual seja conhecido o ren- dimento da bomba. Comumente, mas não necessariamente, $\mathrm{N}_{1}$ é a rotação nominal da bomba. $A$ rotação final $\mathrm{N}_{2}$ é o valor de velocidade com o qual se deseja que a bomba trabalhe. Na ocasião desta pesquisa, $\mathrm{N}_{1}$ é a rotação nominal de cada bomba e $\mathrm{N}_{2}$ é a rotação com a qual a bomba deve operar, a cada hora do dia, de modo que se tenha um custo energético mínimo ao final do ciclo diário.

\section{OTIMIZAÇÃO DO CUSTO ENERGÉTICO}

A otimização energética de um sistema de abastecimento de água envolve muitas possibilidades, tais como o dimensionamento, a expansão, a reabilitação, a confiabilidade hídrica, a redução de perdas e a racionalização do consumo de energia elétrica. Este artigo tem como enfoque a minimização do custo de energia relativo ao funcionamento das bombas num SAA.

Dentre as estratégias que podem ser adotadas para a redução do cus 7 to energético, a alteração das regras operacionais do sistema de bombagem mostra-se muito eficiente, uma vez que não requer aplicação de recursos financeiros e, ainda, proporciona uma economia perceptível em curto prazo. Não obstante, a determinação de regras que minimizam os 
gastos com energia e preservam a qualidade do serviço oferecido aos usuários é um trabalho complicado. Essa atividade envolve diversos fatores, como a consideração do custo tarifário variável e o seu uso de forma eficiente e, ainda, a conservação das variáveis envolvidas dentro de intervalos determinados. Comumente faz-se necessário o uso de modelos que considerem a existência de todos esses fatores (COSTA; CASTRO; RAMOS, 2010).

Conforme estimativas contabilizadas por Balestieri e Vilanova (2015), a adoção de estratégias de eficiência hidráulica e energética é capaz de gerar um potencial de economia de 25 a $50 \%$ no consumo de energia elétrica nos sistemas de abastecimento de água. Segundo Figueiredo (2016), essa consequente economia tem influência direta na redução de despesas operacionais das empresas de saneamento, tendo em vista a responsabilidade ambiental dessas empresas diante de um cenário de escassez hídrica e energética que tem se agravado ao longo dos últimos anos.

No âmbito dos sistemas de abastecimento de água, o consumo de energia elétrica de suas estações elevatórias representa comumente o maior encargo financeiro das entidades gestoras dessas infraestruturas. Diante dessa realidade, muitos pesquisadores têm se dedicado à formulação matemática do problema, objetivando encontrar o modelo ideal de operação dos aparatos elevatórios, ou seja, conseguir o atendimento das necessidades dos consumidores de água com o custo mínimo para a empresa (ALMEIDA, 2010).

Segundo Costa (2010), os problemas de otimização apresentam alguns conceitos largamente empregados e de conhecimento imprescindível. A formulação matemática clássica de um problema dessa natureza é descrita conforme o conjunto de equações a seguir:

$$
\begin{aligned}
& F O=F\left(x_{1}, x_{2}, x_{3}, \ldots, x_{n}\right) \\
& A_{j} \leq g\left(x_{i}\right) \leq B_{j}, \text { com j }=1,2,3, \ldots, \mathrm{J} \\
& x_{i_{-} \min } \leq x_{i} \leq x_{i_{-} \max }
\end{aligned}
$$

Nas Eq. 2, 3 e 4 tem-se que:

- A variável de decisão xi é aquela que é modificada durante o procedimento de busca pela solução ótima. Neste trabalho, trata-se de uma variável real, que representa a velocidade de rotação da bomba. Destaca-se, ainda, que xi assume valores no intervalo [0,1], de modo que significa o percentual da velocidade da bomba em relação à sua rotação nominal.

- As restrições $g\left(x_{i}\right)$ são funções que delimitam as soluções encontradas no sentido de avaliar as suas condições de exequibilidade. No presente trabalho, a restrição feita impõe que o nível do reservatório j mantenha-se sempre entre os valores mínimo $\left(A_{\mathrm{j}}\right)$ e máximo $\left(B_{\mathrm{j}}\right)$.

- A função objetivo (FO) é a função a ser otimizada, no caso, o custo energético durante um ciclo operacional diário é a função que se deseja minimizar.

O procedimento a ser realizado consiste na procura pela configuração ideal de funcionamento das bombas para que se obtenha um custo energético diário mínimo relativo a um SAA. Devido ao grande número de variáveis envolvidas e de restrições peculiares de cada sistema, a execução da referida tarefa pode muitas vezes ser considerada um problema de elevada complexidade. 


\section{PARTICLE SWARM OPTIMIZATION}

A técnica Particle Swarm Optimization (PSO), proposta em 1995 por Russell Eberhart e James Kennedy, tem raízes em duas metodologias de componentes principais. Seus laços mais evidentes estão relacionados com a vida artificial em geral, com o voo dos pássaros, a educação dos peixes e a teoria de enxame em particular. Esse processo otimizador está também relacionado com a computação evolutiva, e tem ligações com algoritmos genéticos e estratégias de evolução.

Segundo Eberhart e Kennedy (1995), pode-se usar otimização por enxame de partículas para resolver muitos dos tipos de problemas que são resolvidos pelos algoritmos genéticos (AGs). $\mathrm{Na}$ otimização pelo PSO, os indivíduos que "voam" próximos a valores ótimos são atraídos por eles e o conhecimento de boas soluções é retido por todas as partículas.

Num enxame, as partículas simbolizam os pássaros, que representam possíveis soluções do problema. A topologia de vizinhança caracteriza o espaço de busca a ser percorrido pelas partículas na busca de soluções ótimas, que equivalem comparativamente aos alimentos caçados pelos pássaros (MEDEIROS, 2005).

Conforme Carvalho (2013), a ideia do PSO é executar um conjunto de operadores com o objetivo de movimentar cada partícula do enxame, por meio de uma busca cooperativa, para a região promissora do espaço de busca. Após cada iteração realizada, é obtido um novo conjunto de soluções. A posição de cada partícula é ajustada pela velocidade, que por sua vez é calculada com base na melhor posição encontrada por uma vizinhança da partícula (componente social) e da melhor posição encontrada pela própria partícula.

O princípio de operação do PSO consiste, inicialmente, na geração aleatória de uma população inicial (enxame de partículas) dentro de um conhecido espaço de busca. A posição de cada indivíduo dentro do espaço representa uma possível solução para o problema de otimização. As partículas se movimentam orientadas por um vetor velocidade associado a elas, que é resultante da soma de três parcelas que o influenciam (SICILIANO, 2007):

- Inércia, que estimula o movimento da partícula no mesmo rumo que inicialmente se movia;

- Memória, que induz a partícula a se mover no sentido do pbest, ou seja, da melhor posição encontrada pela partícula desde o início de sua vida até o presente instante;

- Comparação, que leva a partícula a se movimentar rumo ao gbest, ou seja, ao melhor lugar da região de busca já encontrado pelo enxame.

A Fig. 2 esboça o deslocamento de uma partícula, que anteriormente localizava-se na posição $x_{i}$, para a posição $x_{i+1}$ por efeito do vetor velocidade composto por essas três parcelas.

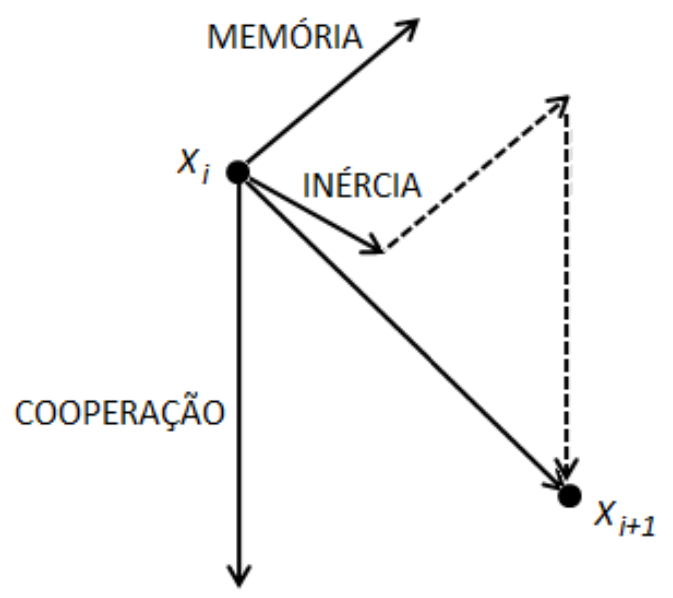

Figura 2 - Movimento de uma partícula no espaço de busca. Fonte: Adaptado de Siciliano (2007).

Após cada iteração, conhecendo-se os valores dos mencionados valores ótimos (pbest e gbest), 
a posição e a velocidade das partículas são atualizadas a partir das equações a seguir:

$$
\begin{aligned}
& v_{i+1}=w \cdot v_{i}+c_{1} \cdot r_{1} \cdot\left(\text { pbest }-p_{i}\right)+c_{2} \cdot r_{2} \cdot\left(\text { gbest }-p_{i}\right) \\
& x_{i+1}=x_{i}+v_{i+1}
\end{aligned}
$$

Nas Eq. 5 e 6, v e $x$ são, respectivamente, a velocidade e a posição de cada partícula, $r_{1}$ e $r_{2}$ são dois números aleatórios pertencentes ao intervalo real $(0,1), w$ é conhecido por parâmetro de inércia e $c_{1}$ e $c_{2}$ são chamados coeficientes de aprendizagem, comumente ambos são iguais a 2. Souza (2006) interpreta que $w, c_{1}$ e $c_{2}$ (que representam papéis social/cognitivo) significam, respectivamente, a confiança da partícula em si mesma, a confiança da partícula na sua experiência e a confiança da partícula nas suas vizinhas.

De acordo com Siciliano (2007), na implementação do algoritmo, a primeira etapa é criar a população inicial de partículas que comporão o enxame com as posições das mesmas. Em seguida, atribui-se a cada partícula um vetor correspondente à sua velocidade inicial. $O$ procedimento de busca do algoritmo permanece atuando, de forma que as velocidades e as posições são atualizadas a cada iteração, até que se alcance um critério de parada (número máximo de gerações percorridas, achado de partícula com determinado grau de aptidão, etc.).

\section{METODOLOGIA}

Para tornar exequível a metodologia ora proposta, desenvolveu-se uma ferramenta computacional no IDE (Integrated Development Environment) Free Pascal Lazarus. A escolha desse ambiente de desenvolvimento e sua referida linguagem se deu devido à sua clareza e facilidade de compreensão, aos seus recursos de programação orientados a objetos e ao seu alto nível de compatibilidade com sistemas operacionais Microsoft Windows. Essa ferramenta encontra-se disponibilizada no endereço https://github.com/jnac1982/UFC12.

O modelo desenvolvido trabalha de maneira acoplada ao simulador hidráulico EPANET. A comunicação entre o software e o EPANET é feita por meio do arquivo epanet2.dll, que é uma biblioteca de vinculo dinâmico (dynamic link-library) pertencente ao kit de ferramentas (toolkit) do EPANET. Essa biblioteca é composta por um conjunto de funções, destinado ao uso de programadores no ambiente Windows.

O modelo proposto atua com o objetivo de otimizar o custo energético em estações elevatórias de SAAs. Para isso, o seu papel é executar o algoritmo de enxame de partículas (PSO) e, por meio da sua rotina, encontrar soluções para o presente problema de otimização. Essas soluções representam as combinações de valores de velocidade de rotação das bombas nas 24 horas do ciclo diário, de modo que cabe ao EPANET avaliar a viabilidade dessas soluções e calcular o custo energético associado a elas.

As etapas do procedimento de otimização são apresentadas na Fig. 3. 


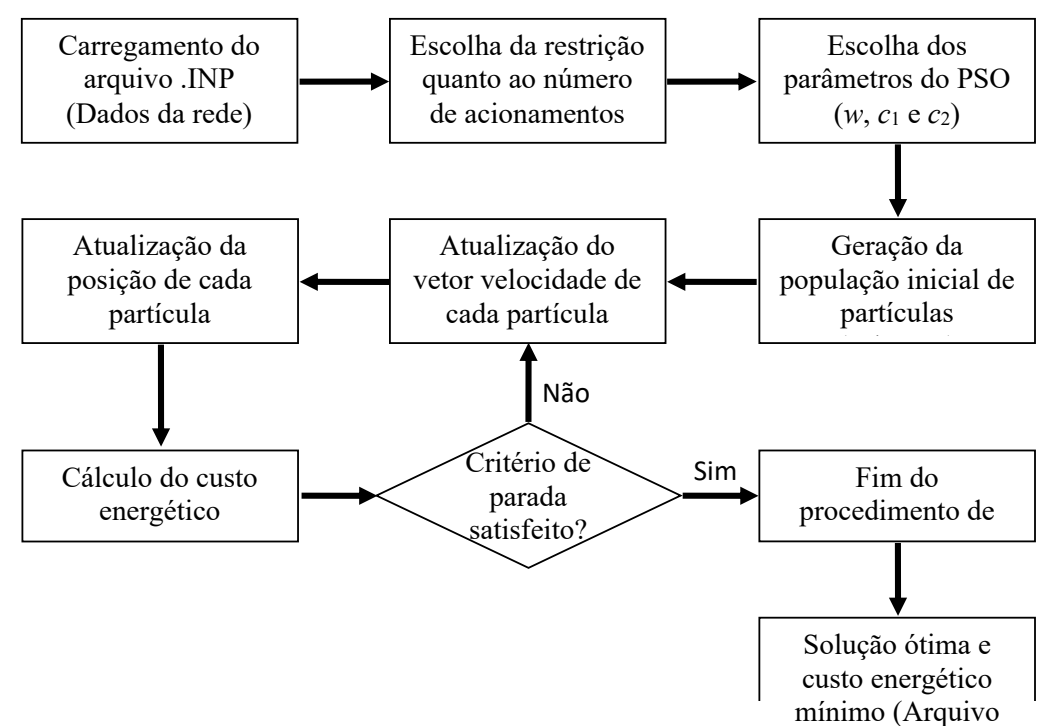

Figura 3 - Fluxograma do processo de busca e otimização realizado pelo modelo proposto.

A execução do software requer o carregamento de um arquivo de entrada, com extensão .INP, contendo as informações da rede a ser analisada. O software importa desse arquivo os dados de quantidade de bombas e reservatórios e de passo de tempo de cálculo hidráulico.

Após o carregamento da rede, pode-se impor uma condição, escolhendo-se um número máximo de acionamentos permitidos para uma mesma bomba (1 a 5) ou optar por uma quantidade livre para esse número. Posteriormente à escolha dessa restrição, devem-se inserir os seguintes parâmetros relativos ao processo de otimização pelo PSO:

- O tempo de execução do programa (em minutos);

- O tamanho da população, que é o número de soluções geradas em cada iteração;

- Os parâmetros w, $c_{1}$ e $c_{2}$ concernentes ao PSO;

- A redução máxima de velocidade de cada bomba, que é o valor abaixo do qual a rotação será considerada nula.
Destaca-se que, para uma rede com $n$ bombas, as soluções geradas são vetores de $24 \cdot n$ coordenadas, que assumem valores no intervalo real $[0,1]$ e representam as velocidades de cada uma dessas bombas a cada hora do dia. 0 valor 0.85 , por exemplo, corresponde a uma bomba operando num certo momento com velocidade equivalente a $85 \%$ da sua rotação nominal. No caso de uma rede com 3 bombas, cada vetor solução é, portanto, composto por 72 coordenadas, sendo as três primeiras dessas as velocidades das bombas 1, 2 e 3, nessa ordem, na primeira hora do ciclo diário. As próximas três coordenadas são as velocidades das respectivas bombas na segunda hora e, assim por diante, até a $24^{\mathrm{a}}$ hora.

Inicia-se, então, a execução do procedimento de busca e otimização, que consiste na procura por soluções viáveis (que atendem à restrição imposta) e pela solução ótima (que tem custo energético mínimo). Conforme a Fig. 3, o início desse processo se dá com a criação de uma população inicial de partículas. A cada vetor solução (posição de uma partícula), corresponde um vetor velocidade de mesmo tamanho. A cada iteração, essas velo- 
cidades são atualizadas pela Eq. 5 e, em seguida, as posições são atualizadas pela Eq. 6.

Em seguida, o custo energético associado a cada uma dessas soluções é calculado pelo EPANET e, a cada iteração, são armazenadas as informações referentes ao número de soluções viáveis contabilizadas e à melhor solução encontrada até o momento. $O$ algoritmo PSO prevê a repetição desse processo até que seja atingido um critério de parada. No presente trabalho, escolheuse para esse critério o tempo predefinido para a execução do programa.

Finalmente, gera-se um arquivo em formato TXT com o total de gerações percorridas e de soluções viáveis registradas. Além disso, esse arquivo contém a solução ótima e o custo energético mínimo ao longo das gerações percorridas.

Salienta-se que na etapa do cálculo do custo energético, propôs-se uma correção do valor do rendimento final da bomba, calculado para quando a sua velocidade de rotação é variada. Marchi e Simpson (2013) afirmam que o simulador hidráulico EPANET permanece a utilizar o rendimento da bomba relativo à sua rotação nominal mesmo quando há alteração na velocidade. Dessa forma, torna-se impreciso o cálculo da potência, da energia consumida e do custo energético. Essa correção do rendimento foi realizada com o auxílio da Eq. 1.

\section{APLICAÇÃO DO MODELO PROPOSTO}

O modelo computacional ora proposto foi aplicado a um estudo de caso hipotético, cuja escoIha foi feita por se tratar de uma rede já utilizada em outros trabalhos de otimização. Em pesquisas anteriores, o custo energético mínimo desse estudo de caso foi determinado por meio de outros algoritmos de otimização, como o algoritmo genético simples e um algoritmo genético híbrido no trabalho de Costa (2010), e ainda, o al- goritmo genético $A G_{60}$ e o enxame de partículas para uma variável binária no trabalho de Hortegal Filha (2017). Desse modo, pôde-se comparar o desempenho dos métodos aplicados.

Salienta-se que no presente trabalho o algoritmo de otimização PSO é aplicado a uma variável de decisão contínua (velocidade relativa de rotação das bombas), diferentemente das outras pesquisas, nas quais o estado das bombas (ligado/desligado) é uma variável de decisão binária. Desta forma, a função objetivo é o custo energético diário, expresso pela Eq. 7.

$$
C E=\sum_{n=1}^{N_{B}} \sum_{t=1}^{24} C_{n t} \cdot E_{n t}
$$

$\mathrm{Na}$ Eq. 7, destaca-se que NB é a quantidade de bombas, $n$ é o número de uma bomba $\left(1 \leq n \leq N_{B}\right)$, t é a hora do dia $(1 \leq t \leq 24)$, Cnt é o custo tarifário (\$/kWh), Ent é a energia gasta na bomba $n$ na hora t (kWh) e CE é o custo energético diário (\$).

Convém ressaltar que, na presente pesquisa, o único custo considerado no problema de otimização foi o custo energético relativo ao funcionamento das bombas de um SAA. Desse modo, não se analisaram estratégias para a minimização de custos de outras naturezas, como por exemplo, instalação e manutenção das bombas.

A rede escolhida é um sistema hipotético criado por Walski et al. (1987) e atualizada por Rao e Salomons (2007). Conforme a Fig. 4, a rede é composta por 3 reservatórios com níveis variáveis (cada um com $5 \mathrm{~m}$ de altura), 3 bombas, 41 tubulações e 19 nós, sendo que três destes admitem valores críticos de pressão (nós 55, 90 e 170). Destaca-se que para os três reservatórios a cota piezométrica mínima é igual a $66.53 \mathrm{~m}$, enquanto a máxima equivale a $71.53 \mathrm{~m}$. Com relação aos nós críticos, as pressões mínimas nos mesmos assumem os seguintes valores: $51 \mathrm{mca}$ (nó 90), 42 mca (nó 55) e 30 mca (nó 170). 


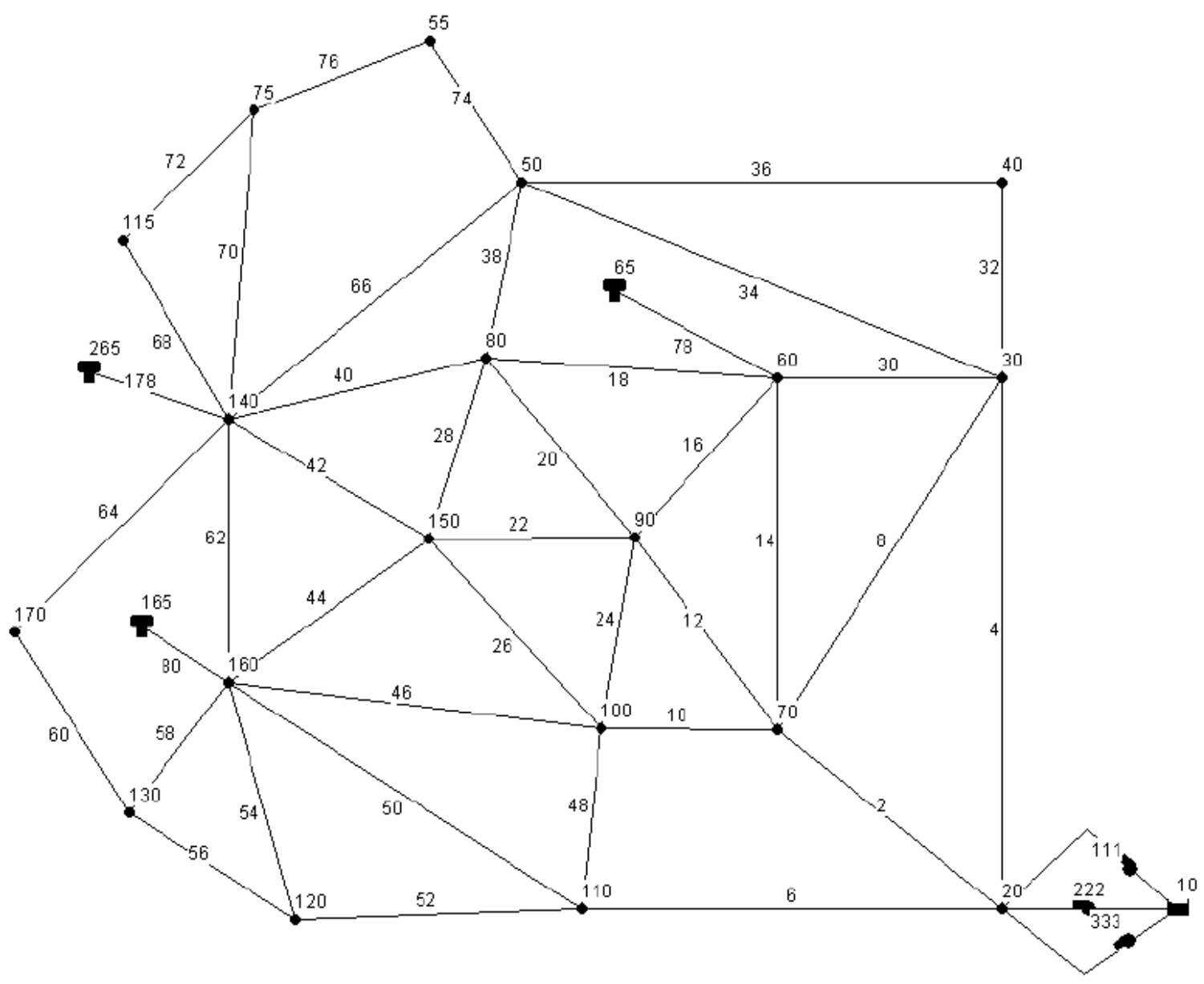

Figura 4 - Topologia da rede estudo de caso. Fonte: Rao e Salomons (2007).

Nesta pesquisa, adotou-se o mesmo custo tarifário energético horário utilizado por Rao e Salomons (2007). No decorrer do ciclo diário, a tarifa assume três valores distintos $(0.1814 \$ / \mathrm{kWh}$, $0.3528 \$ / \mathrm{kWh}$ e $0.8097 \$ / \mathrm{kWh}$ ), distribuídos em quatro intervalos. O horário de tarifa mais elevada está compreendido entre a $18^{\mathrm{a}}$ e a $21^{\mathrm{a}}$ hora do dia. Esses valores de custo tarifário foram criados por esses autores para serem aplicados ao caso hipotético estudado por eles. Assim, os referidos autores escolheram não definir a moeda relativa a essa tarifa e, desse modo, neste trabalho manteve-se a opção de escolha por uma moeda indefinida.

\section{RESULTADOS}

Relativamente à aplicação do modelo desenvolvido, salienta-se que sobre as restrições impostas no início da aplicação, foi pressuposto que a quantidade máxima de acionamentos diários é livre e, ainda, considerou-se a hipótese que, para cada reservatório, o nível final deve ser igual ou superior ao nível inicial. Essa última restrição, relativa ao nível dos reservatórios, é utilizada por alguns autores (PEDROSA, 2006; CUNHA, 2009; COSTA, 2010) em seus trabalhos de otimização. $O$ intuito da aplicação dessa medida restritiva é que, ao final do ciclo operacional, o sistema possa garantir a continuidade da operação em períodos subsequentes. 
O procedimento de busca realizado teve tempo de execução igual a 1 hora. Com relação à escoIha dos parâmetros iniciais de busca pelo PSO, utilizaram-se os seguintes valores:

- Redução máxima de velocidade igual de 70\%;

- Tamanho da população de cada geração igual a 100 indivíduos;

- Parâmetro de inércia w igual a 1.4;

- Coeficientes $c_{1}$ e $c_{2}$ ambos iguais a 2 .
Após a conclusão desse primeiro procedimento, verificou-se que foram encontradas ao todo 11 soluções viáveis inseridas num conjunto limitado de 3716 gerações criadas e vasculhadas. 0 valor do custo energético mínimo diário, relativo à melhor solução, foi igual a \$3735.55. Além dos valores do custo tarifário energético considerado, a Fig. 5 exprime as configurações de funcionamento diário de cada uma das bombas relativas à solução ótima.

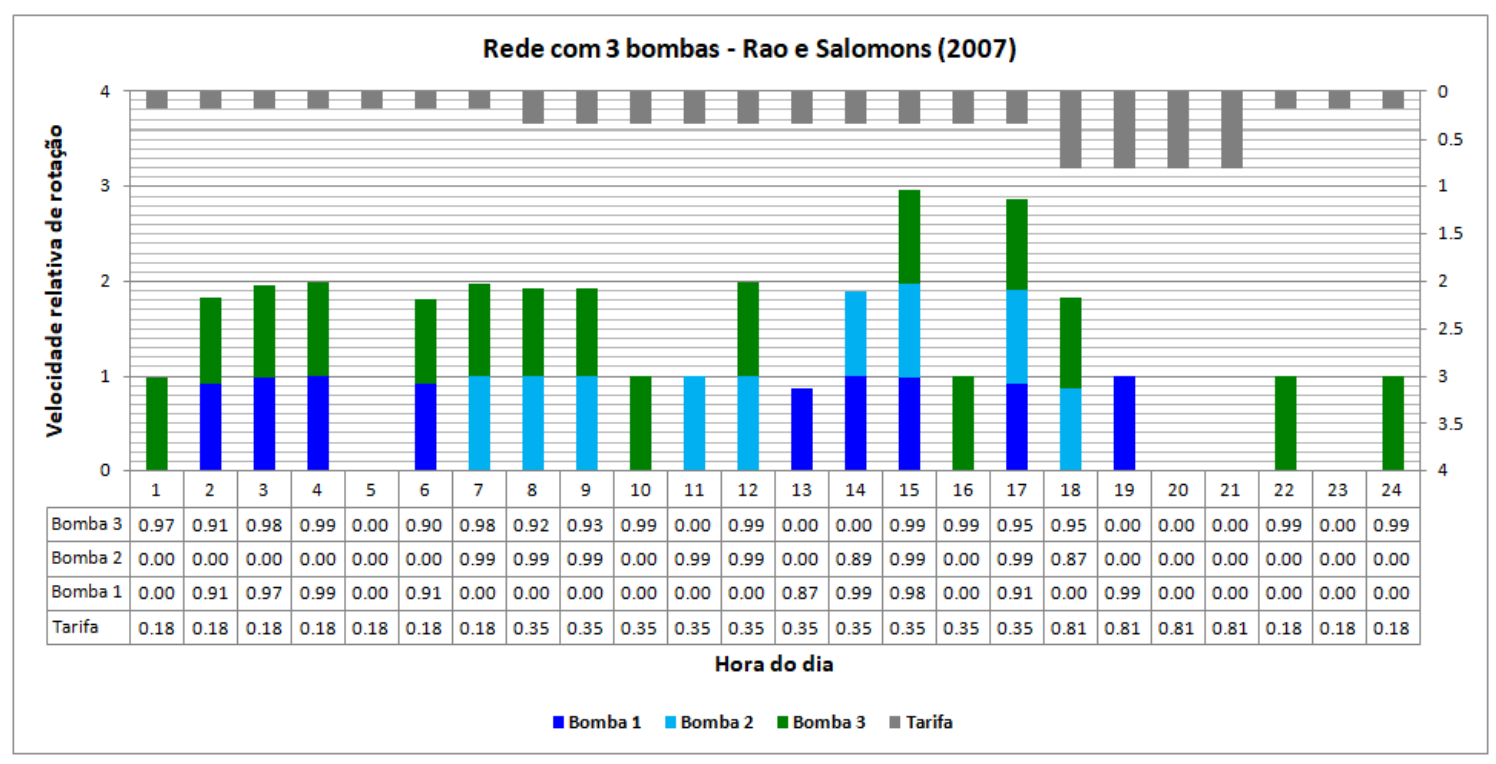

Figura 5 - Solução ótima e tarifa energética.

Conforme pode se verificar na Fig. 5, os perfis de velocidade das bombas aparentam se adequar ao valor da tarifa energética. Observa-se que a velocidade de qualquer uma das bombas, em qualquer instante, na configuração ótima de operação, assume um valor igual ou superior a $87 \%$ da sua rotação nominal ou é igual a zero (bomba desligada).

No que se refere ao período em que a tarifa energética é menor, identifica-se que, com as exceções da quinta e da vigésima terceira hora do dia, em todos os outros instantes há alguma bomba que se encontra em atividade. Sobre o ínterim em que a tarifa é mais elevada, constata-se que no segundo momento desse intervalo (19a hora) duas bombas se mantêm desligadas e em dois momentos ( $20^{\mathrm{a}}$ e $21^{\mathrm{a}}$ horas), as três bombas permanecem inoperantes.

A matriz abaixo do gráfico da Fig. 5 representa a configuração diária de velocidades das bombas relativa ao consumo energético ótimo. A primeira linha representa os valores de rotação ao longo do dia para a bomba 1, a segunda refere-se à bomba 2 e a terceira, por sua vez, refere-se à 
bomba 3.0 acionamento de uma bomba durante o ciclo pode ser identificado a partir de uma hora em que ela estava desligada (velocidade nula) e na hora seguinte passou a operar com valor não nulo de velocidade. Assim, observa-se que a solução ótima prevê as seguintes quantidades de acionamentos diários: 5 para a bomba $1\left(2^{\mathrm{a}}, 6^{\mathrm{a}}\right.$, $13^{\mathrm{a}}, 17^{\mathrm{a}}$ e $19^{\mathrm{a}}$ horas), 4 para a bomba $2\left(7^{\mathrm{a}}, 11^{\mathrm{a}}\right.$, $14^{\mathrm{a}}$ e $17^{\mathrm{a}}$ horas) e 5 para a bomba $3\left(6^{\mathrm{a}}, 12^{\mathrm{a}}, 15^{\mathrm{a}}\right.$, $22^{\mathrm{a}}$ e $24^{\mathrm{a}}$ horas).

A Fig. 6 exibe o padrão diário de variação dos níveis dos três reservatórios que compõem o SAA em análise. Observa-se uma semelhança no comportamento da altura da superfície d'água nos mesmos. Desse modo, na quase totalidade do período de um dia, quando um reservatório se encontra em processo de enchimento, os outros dois também estão e, de maneira similar, acontece o esvaziamento deles.

O reservatório RNV 65, que se encontra mais próximo às bombas, possivelmente devido a esse fato, tem seu nível mais elevado que os demais ao longo do dia. Ademais, pode-se identificar que o seu nível cresce e decresce mais rapidamente quando em comparação aos outros dois reservatórios. Destaca-se, ainda, que conforme a restrição inicial, o nível final de cada um deles é superior ou igual ao inicial, não obstante essa diferença seja muito pequena nos casos dos reservatórios RNV 165 e RNV 265, situados mais distantes das bombas.

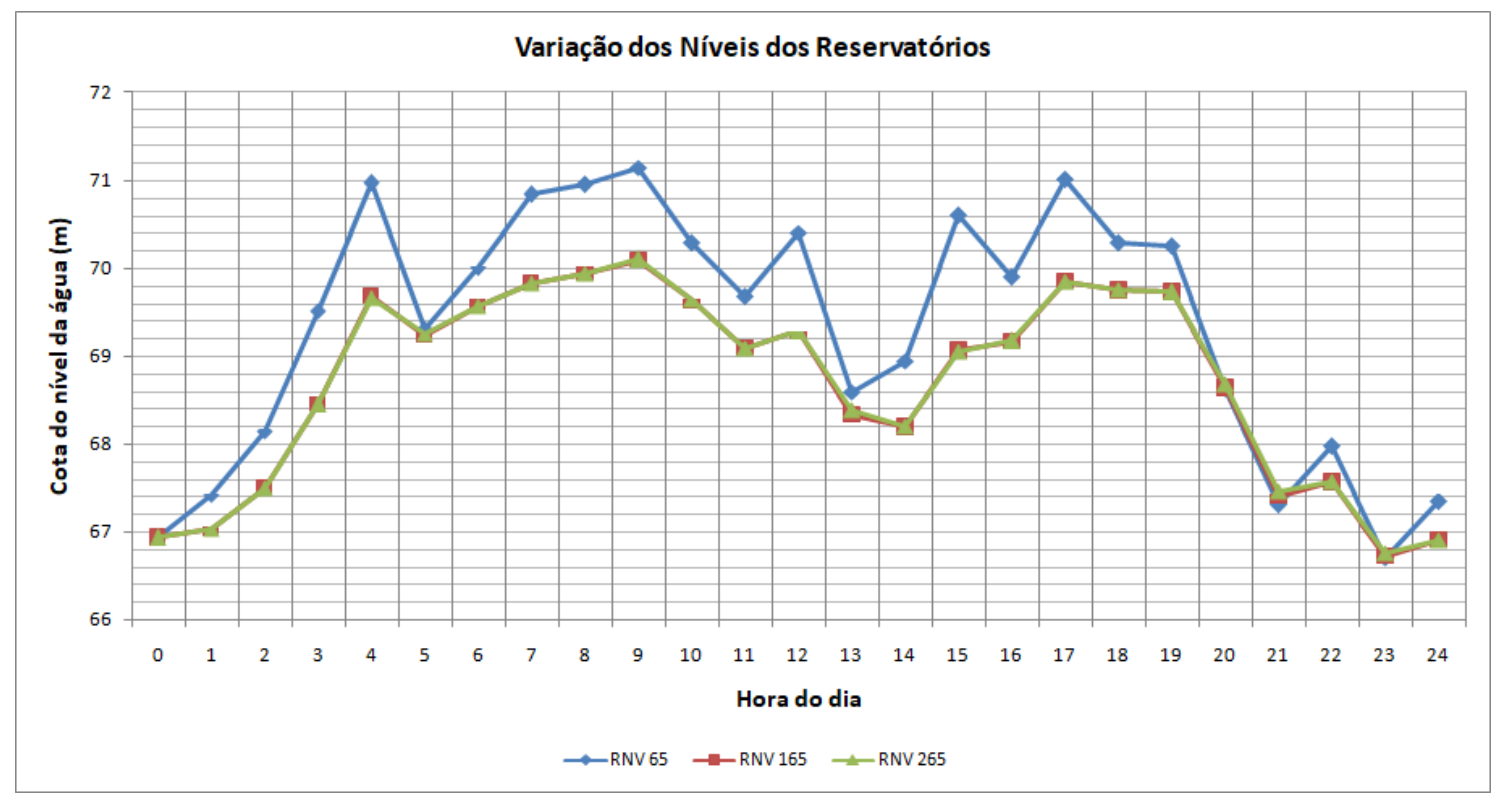

Figura 6 - Variação dos níveis dos reservatórios.

De maneira coerente com a configuração diária de funcionamento das bombas do sistema, há um decaimento mais acentuado nos níveis dos três reservatórios ao final da quarta hora do dia e no período compreendido entre a décima nona e a vigésima primeira hora. Tal fato ocorre nos momentos em que as três bombas mantêm-se desligadas, devido ao valor mais caro da tarifa no segundo intervalo mencionado. 


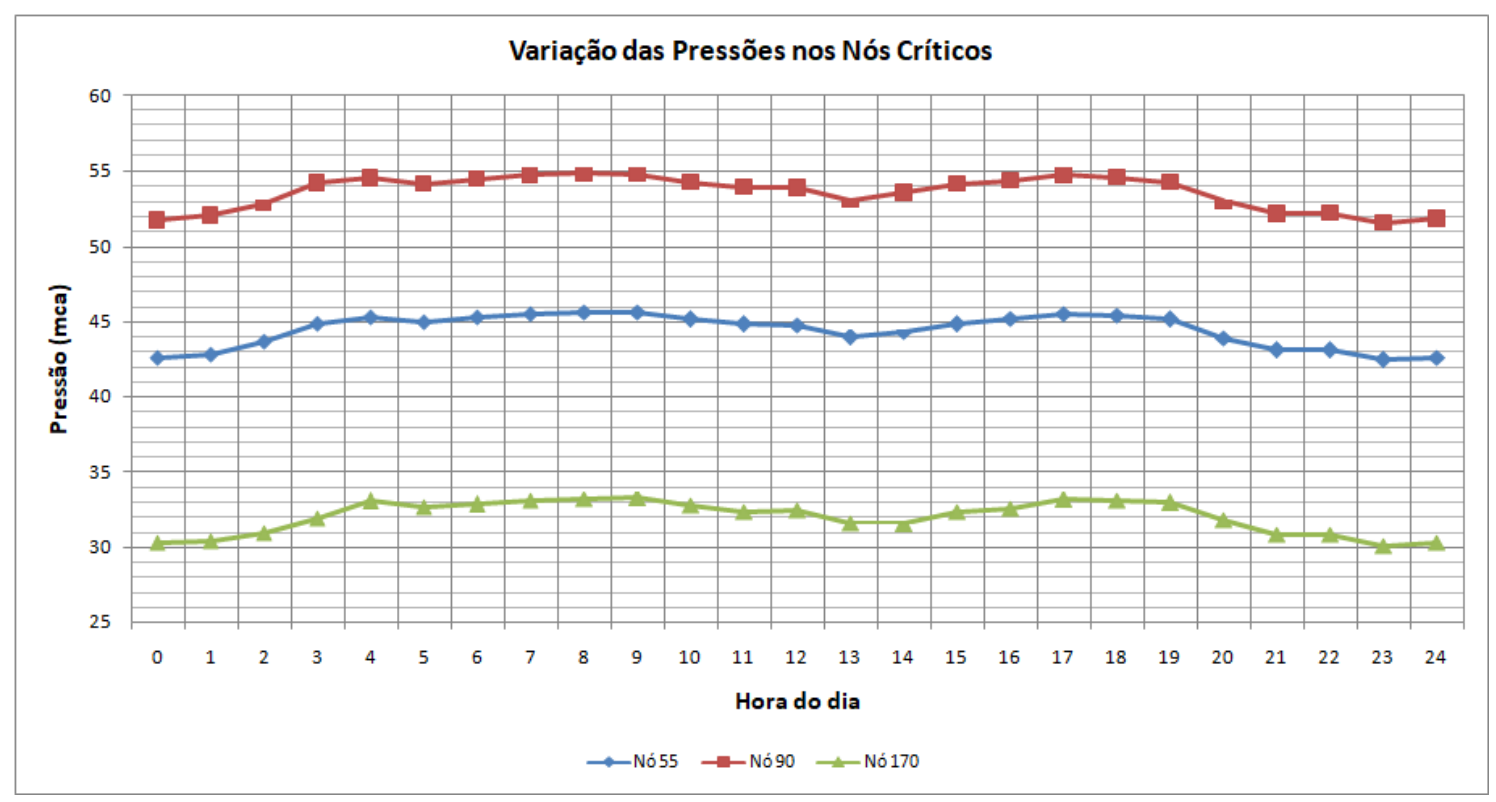

Figura 7 - Variação das pressões nos nós críticos.

A Fig. 7 expressa a oscilação diária das pressões nos nós críticos para a solução ótima encontrada. Conforme se pode observar, evidencia-se que em nenhum momento a pressão em qualquer um dos referidos nós assumiu um valor inferior ao limitante mínimo indicado.

Pode-se observar que o instante em que a pressão mais se aproximou do seu valor mínimo foi o mesmo nos três nós críticos. Esse fato ocorreu no final da $23^{\mathrm{a}}$ hora do dia, quando as pressões nos nós 55,90 e 170 apresentaram valores iguais a 42.39 m, 51.48 m e 30.10 m, respectivamente. Destaca-se que a ocorrência de mínima pressão nesses nós se deu no mesmo instante em que os níveis dos reservatórios foram os menores observados. Ambos os fatos se justificam pelo desligamento concomitante das três bombas na $23^{\mathrm{a}}$ hora.

Finalmente, comparou-se o desempenho do algoritmo de otimização utilizado neste trabalho (PSO aplicado a uma variável real) com o desem- penho de outras técnicas aplicadas à mesma rede de estudo em pesquisas predecessoras.

Nos experimentos realizados por Costa (2010) para o SAA em questão, o referido autor encontrou, por meio de dois algoritmos otimizadores distintos (AGS e AGH), uma mesma solução ótima, cujo custo energético equivale a $\$ 3606.00$. Os métodos de busca especificados divergiram apenas com relação ao tempo necessário para que a solução ótima fosse detectada.

Hortegal Filha (2017), com o uso do algoritmo genético $A G_{60}$, encontrou uma solução de alto valor de custo energético ótimo equivalente a \$3958.40. Entretanto, por meio do outro método de busca empregado pela mencionada autora, o processo de otimização foi mais bem-sucedido, uma vez que com o uso do PSO binário foi encontrada uma solução ótima de custo igual a \$3601.05. 
Tabela 1- Custo ótimo conforme a técnica de otimização.

\begin{tabular}{|c|c|c|}
\hline $\begin{array}{c}\text { Algoritmo } \\
\text { Otimizador }\end{array}$ & $\begin{array}{c}\text { Custo diário da } \\
\text { Solução Ótima (\$) }\end{array}$ & $\begin{array}{c}\text { Tempo de } \\
\text { Processamento (min) }\end{array}$ \\
\hline AGS & 3606.00 & 141.77 \\
\hline AGH & 3606.00 & 11.23 \\
\hline AG $_{60}$ & 3958.40 & - \\
\hline PSO Binário & 3601.05 & - \\
\hline PSO Real & 3735.55 & 14.65 \\
\hline
\end{tabular}

Na presente investigação, o algoritmo PSO real apresentou uma vantagem considerável em relação ao método $\mathrm{AG}_{60}$, uma vez que encontrou uma solução ótima de custo energético mais barato. No entanto, se comparado aos algoritmos AGS, AGH e PSO binário, o PSO real não se mostrou mais vantajoso, pois a solução ótima encontrada por ele possui valor de custo energético superior aos demais. Não obstante, convém destacar uma característica favorável do PSO real em relação ao AGS no que se refere à rapidez na detecção da solução ótima. Nota-se que o AGS demorou 141.77 minutos para chegar ao custo energético mínimo, enquanto o PSO real demandou apenas 14.65 minutos para o mesmo propósito.

\section{CONCLUSÕES}

0 presente trabalho teve por objetivo o desenvolvimento de uma rotina computacional que pudesse simular o comportamento de bombas hidráulicas de velocidade de rotação variável e, ainda, de maneira acoplada ao EPANET, encontrar padrões diários de velocidade associados a um custo energético ótimo. A rotina proposta foi aplicada a um SAA hipotético, executando a busca por configurações diárias de funcionamento de suas bombas, de modo a se obter uma solução de custo energético mínimo. A proposição central da pesquisa consistiu na aplicação do algoritmo de enxame de partículas (PSO) à realização dessa tarefa.

A aplicação da ferramenta computacional proposta ao presente estudo de caso evidenciou o êxito do algoritmo PSO frente ao problema de otimização do custo energético de bombas de velocidade de rotação variável. A eficiência do modelo proposto pôde ser constatada diante do sucesso na busca por soluções viáveis (que atendem às restrições preestabelecidas) e na determinação da solução ótima (que implica em um custo energético mínimo). Diante de seu desempenho favorável, foi possível constatar a adequabilidade do PSO a um problema de otimização com variável de decisão real.

Convém ressaltar que a complexidade do problema ora abordado é consideravelmente superior à das situações que envolvem bombas de rotação fixa, nas quais a variável de decisão é binária. Portanto, dada essa complexidade, que envolve o controle da velocidade das bombas e o consequente ajuste de seu funcionamento à demanda sazonal diária de vazão e pressão, é incontestável a participação do algoritmo otimizador na solução desse problema.

Enfim, a metodologia apresentada no presente trabalho contribui no fornecimento de suporte à determinação de estratégias operacionais ótimas de SAAs, visando sua eficiência energética. Desta forma, conclui-se que é plenamente concebível a adoção dos procedimentos ora apresentados, por parte das empresas de abastecimento de água, como ferramenta de auxílio à tarefa dos operadores de seus sistemas de bombeamento.

\section{CONTRIBUIÇÃO DOS AUTORES}

Concepção do Artigo: Costa JNA e Castro MAH; Metodologia: Costa JNA e Costa LHM; Desenvolvimento da Ferramenta Computacional: Costa LHM; Discussão dos Resultados: Costa LHM e Barbosa JMC; Redação - Primeira Versão: Costa JNA; Redação - Revisao \& Edição: Costa JNA e Costa LHM. 


\section{REFERÊNCIAS}

ALEGRE, H.; COELHO, S. T.; ALMEIDA, M. C.; VIEIRA, P. Controlo de perdas de água em sistemas públicos de adução e distribuição. 328 p. Instituto Regulador de águas e Resíduos, Instituto da Água, Laboratório nacional de Engenharia Civil, Portugal, 2005.

ALMEIDA, P. L. C. Optimização da Operação de uma Estação Elevatória. Minimização do Custo da Energia Respeitando Critérios de Qualidade da Água. 156 p. Dissertação (Mestrado Integrado em Engenharia Civil) - Faculdade de Engenharia, Universidade do Porto, Porto, 2010.

BALESTIERI, J.A.P, VILANOVA, M.R.N. Exploring de water-energy nexus in Brasil: The electricity use for water supple. Revista Energy, n. 85, p. 415-432, abr. 2015. https://doi.org/10.1016/j. energy.2015.03.083

BAPTISTA, M. B.; COELHO, M. L. P. Fundamentos de Engenharia Hidráulica. $3^{\mathrm{a}}$ ed. revista e ampliada - Belo Horizonte: Editora UFMG, 2010. 480p.

BORGES, A. Apostila Máquinas Hidráulicas - Bombas. Universidade Federal do Rio Grande do Sul. 2011.

CARVALHO, A. B. Novas estratégias para otimização por nuvem de partículas aplicadas a problemas com muitos objetivos. 218 p. Tese (Doutorado) - Universidade Federal do Paraná, Curitiba, 2013.

COSTA, L. H. M. Utilização de um algoritmo genético híbrido na operação de sistemas de abastecimento de água com ênfase na eficiência energética. 146 p. Tese (Doutorado) - Universidade Federal do Ceará, Fortaleza, 2010.

COSTA, L. H. M.; CASTRO, M. A. H.; RAMOS, H. Utilização de um algoritmo genético híbrido para operação ótima de sistemas de abastecimento de água. Reg. ABES v.15, n. 2, p. 187-196, abr./jun. 2010. https://doi.org/10.1590/S1413-41522010000200011

COSTA, J. N. A.; CASTRO, M. A. H.; COSTA, L. H. M.; BARBOSA, J. M. C. New formula proposal for the determination of variable speed pumps efficiency. Brazilian Journal of Water Resources, v. 23, e44, p. 1-11, 2018. https://doi.org/10.1590/23180331.231820180003

CUNHA, A. A. R. Otimização energética em tempo real da operação de sistemas de abastecimento de água. 179 p. Dissertação (Mestrado) - Universidade de São Paulo, São Carlos, 2009.

EBERHART, R. KENNEDY, J. A new optimizer using particle swarm theory. In: Sixty International Symposium on Micro Machine and Human Science, Nagoya, oct., 1995. https://doi. org/10.1109/MHS.1995.494215

FIGUEIREDO, P. O. S. D. Estruturação de Método para Avaliação de Desempenho Energético de Estações Elevatórias de Sistemas de Abastecimento de Água. 125 p. Dissertação (Mestrado em Engenharia de Recursos Hídricos e Ambiental) - Universidade Federal do Paraná, Curitiba, 2016.
HORTEGAL FILHA, M. S. R. Análise de algoritmos evolucionários aplicados à otimização de estação elevatória em redes de abastecimento de água. 108 p. Tese (Doutorado) - Universidade Federal do Ceará, Fortaleza, 2017.

KURITZA, J. C. Metodologia para Avaliação da Eficiência Energética de Sistemas de Bombeamento de Água com Velocidade de Rotação Variável. 160 p. Tese (Doutorado em Engenharia) Universidade Federal do Rio Grande do Sul, Porto Alegre, 2017.

KURITZA, J. C.; CAMPONOGARA, G.; CASTIGLIO, G. S.; KEMPKA, M.; BRAVO, J. M.; MARQQUES, M. G. Utilização do Algoritmo SCE-UA para Obtenção de Regra de Operação Otimizada de Sistema de Bombeamento. In: XXII SBRH - SIMPÓSIO BRASILEIRO DE RECURSOS HÍDRICOS, Florianópolis, nov. 2017. Anais...

LINSINGEN, I. V. Fundamentos de Sistemas Hidráulicos. Editora da UFSC. 399 p. Santa Catarina, 2001.

MARCHI, A.; SIMPSON, A. R. Correction of the EPANET inaccuracy in computing the efficiency of variable speed pumps. Journal of Water Resources Planning and Management, v. 139, n. 4, p. 456-459, jul. 2013. https://doi.org/10.1061/(ASCE)WR.19435452.0000273

MARCHI, A.; SIMPSON, A. R; ERTUGRUL, N. Assessing variable speed pump efficiency in water distribution systems. Engineering and Science, v. 5, n. 1, p. 15-21, jul. 2012. https://doi. org/10.2166/aqua.2017.086

MEDEIROS, J. A. C. C. Enxame de partículas como ferramenta de otimização em problemas complexos de engenharia nuclear. 108 p. Tese (Doutorado) - Universidade Federal do Rio de Janeiro, Rio de Janeiro, 2005

PEDROSA, L. A. F. Um modelo de operação de sistemas adutores de abastecimento de água com vistas a minimização dos custos energéticos. 160 p. Tese (Doutorado) - Universidade Federal de Campina Grande, Campina Grande, 2006.

RAO, Z.; SALOMONS, E. Development of a Real-Time, Near Optimal, Control Process for Water-Distribution Networks. Journal of Hydroinformatics, v. 09.1, p. 25-38, 2007. https://doi. org/10.2166/hydro.2006.015

REIS, R. L. Eficiência Energética em Processos de Bombeamento D’Água. 90 p. Universidade Federal da Bahia, Salvador, 2010.

RODRIGUES, W. Critérios para o uso eficiente de inversores de frequência em sistemas de bombeamento de água. 2007. 208 p. Tese (Doutorado em Engenharia Civil) - Universidade Estadual de Campinas, Campinas, 2007.

SCHMIDLIN JR., C. R.; MOREIRA, A. B.; CAVALCANTE NETO, T. N.; PITOMBEIRA, E. S.; PONTES, R. S. T. Eficiência Energética em Sistemas de Bombeamento. In: VII CONFERÊNCIA INTERNACIONAL DE APLICAÇÕES INDUSTRIAIS - INDUSCON, Recife, jan. 2006. Anais... 
SICILIANO, A. V. Algoritmos Genéticos e Particle Swarm Optimization e suas aplicações problemas de Guerra Eletrônica. Diretoria de Sistemas de Armas da Marinha (DSAM). Rio de Janeiro, RJ, 2007.

SOUZA, G. R. Uma Abordagem por Núvens de Partículas para Problemas de Otimização Combinatória. 80 p. Dissertação (Mestrado) - Universidade Federal do Rio Grande do Norte, Natal, 2006.
WALSKI, T. M.; BRILL Jr., E. D.; GESSLER, J.; GOULTER, I. C.; JEPPSON, R. M.; LANSEY, K.; LEE, H.; LIEBMAN, J. C.; MAYS, L.; MORGAN, D. R.; ORMSBEE, L. Battle of the network models: epilogue. Journal of Water Resources Planning and Management, ASCE, Vol. 113, No. 2, p. 191-203, mar. 1987. https://doi.org/10.1061/ (ASCE)0733-9496(1987)113:2(191) 